\title{
The interpretation of water emission from dense interstellar clouds
}

\author{
D. R. Poelman ${ }^{1,2}$, M. Spaans ${ }^{1}$, and A. G. G. M. Tielens ${ }^{3,1}$
}

\author{
1 Kapteyn Astronomical Institute, PO Box 800, 9700 AV Groningen, The Netherlands \\ e-mail: D.R.Poelman@astro.rug.nl \\ 2 SRON Netherlands Institute for Space Research, Landleven 12, 9747 AD Groningen, The Netherlands \\ 3 NASA Ames Research Center, MS245-3, Moffett Field, CA 94035, USA
}

Received 4 August 2006 / Accepted 11 January 2007

\section{ABSTRACT}

\begin{abstract}
Context. Existing SWAS observations and future HIFI/Herschel data require a clear sense of the information content of water emission and absorption lines.

Aims. We investigate whether the ground-state transition of ortho- $\mathrm{H}_{2} \mathrm{O}\left(1_{10} \rightarrow 1_{01}\right)$ at $557 \mathrm{GHz}$ can be used to measure the column density throughout an interstellar cloud.

Methods. We make use of a multi-zone escape probability code suitable for treating molecular line emission.

Results. For low abundances, i.e., $X\left(\mathrm{H}_{2} \mathrm{O}\right) \lesssim 10^{-9}$, the intensity of the $1_{10} \rightarrow 1_{01}$ transition scales with the total column density of $\mathrm{H}_{2}$. However, this relationship breaks down with increasing abundance, i.e., optical depth, due to line trapping and - for $T_{\text {dust }} \gtrsim 25 \mathrm{~K}$, $X\left(\mathrm{H}_{2} \mathrm{O}\right) \lesssim 10^{-8}$ and $n \sim 10^{4} \mathrm{~cm}^{-3}-$ absorption of the dust continuum.

Conclusions. An observed decline in intensity per column density, expected if $\mathrm{H}_{2} \mathrm{O}$ is a surface tracer, does not necessarily mean that the water is absent in the gas phase at large column densities, but can be caused by line trapping and subsequent collisional deexcitation. To determine the amount of water vapor in the interstellar medium, multiple line measurements of optically thin transitions are needed to disentangle radiative transfer and local excitation effects.
\end{abstract}

Key words. ISM: molecules - radiative transfer - ISM: dust, extinction

\section{Introduction}

The launch of the Submillimeter Wave Astronomy Satellite (SWAS, Melnick et al. 2000) made it possible to observe emission from the ground-state transition $\left(1_{10} \rightarrow 1_{01}\right)$ of ortho- $\mathrm{H}_{2}^{16} \mathrm{O}$ and its isotopomer ortho- $\mathrm{H}_{2}^{18} \mathrm{O}$, to determine the abundance of water vapor, i.e., column density $N_{\mathrm{o}-\mathrm{H}_{2} \mathrm{O}}$, in various regions in the interstellar medium, e.g., dense and diffuse interstellar gas clouds, circumstellar envelopes, planetary atmospheres, and comets (e.g., Melnick et al. 2000; Snell et al. 2000a,b; Encrenaz et al. 1995; Bergin et al. 2000a; Gurwell et al. 2000; Melnick et al. 2000). In the future, the Heterodyne Instrument for the Far Infrared (HIFI) on Herschel will observe even more transitions of ortho- and para- $\mathrm{H}_{2} \mathrm{O}\left(\mathrm{o} / \mathrm{p}-\mathrm{H}_{2} \mathrm{O}\right)$ like $2_{12} \rightarrow 1_{01} \quad(1669.904 \mathrm{GHz}), 2_{21} \rightarrow 2_{12} \quad(1661.015 \mathrm{GHz})$, $3_{03} \rightarrow 2_{12} \quad(1716.774 \mathrm{GHz}), \quad 3_{12} \rightarrow 3_{03} \quad(1097.357 \quad \mathrm{GHz})$, $3_{21} \rightarrow 3_{12}(1162.910 \mathrm{GHz}), 1_{11} \rightarrow 0_{00}(1113.342 \mathrm{GHz}), 2_{02} \rightarrow 1_{11}$ $(967.924 \mathrm{GHz}), \quad 2_{11} \rightarrow 2_{02} \quad(752.029 \mathrm{GHz})$, and $2_{20} \rightarrow 2_{11}$ $(1228.801 \mathrm{GHz})$, with some in absorption while others in emission.

One of the main goals of the SWAS mission is to determine where and whether $\mathrm{H}_{2} \mathrm{O}$ and $\mathrm{O}_{2}$ are the major reservoirs of oxygen through the interstellar medium. SWAS observations have determined the gaseous water abundance in warm dense gas $\left(T \gtrsim 300 \mathrm{~K}\right.$ and $n\left(\mathrm{H}_{2}\right) \gtrsim 10^{3} \mathrm{~cm}^{-3}$ ) to be $10^{-5}$ relative to $\mathrm{H}_{2}$, in good agreement with chemical models for such conditions. However, in cold $(T \lesssim 30 \mathrm{~K})$, dense clouds the abundance of gaseous water is $\sim 100$ to 1000 times below the predictions of cold-cloud gas-phase chemical models. It has been suggested that - toward cold clouds - gaseous $\mathrm{H}_{2} \mathrm{O}$ exists only near the cloud surface. Indeed, because closer to the surface than an $A_{\mathrm{V}}$ of a few mag, $\mathrm{H}_{2} \mathrm{O}$ is photo-dissociated by the ambient galactic UV field. Deeper into the cloud, i.e., $A_{\mathrm{V}}$ of 4-8 mag (depending on density and UV intensity), $\mathrm{H}_{2} \mathrm{O}$ may rapidly deplete onto dust grains (Bergin et al. 2000b; Charnley et al. 2001; Viti et al. $2001)$. Although the derivation of the column density from $a b$ sorption observations is straightforward (column density is simply proportional to the optical depth in the line, see Plume et al. 2004), this is not the case for emission observations. The analysis to determine the $\mathrm{H}_{2} \mathrm{O}$ abundance now crucially depends on the physical properties of the gas through the collisional rate coefficients. Therefore, accurate constraints on the gas densities and temperatures are needed.

Extensive SWAS observations of the Orion A molecular cloud show that gaseous $\mathrm{H}_{2} \mathrm{O}$ correlates with $\mathrm{CN}$, a surface tracer, rather than with $\mathrm{C}^{18} \mathrm{O}$, a volume tracer (Melnick \& Bergin 2005). This result has been interpreted as evidence that gaseous water resides only near the surface. However, caution is needed when relying purely on single transition observations to draw such a conclusion in view of the complex rotational level structure of the $\mathrm{H}_{2} \mathrm{O}$ molecule. In particular, there is a fundamental difference between an optically thin and an effectively optically thin line. The latter case implies a strong coupling between line photons and water molecules. A full radiative transfer calculation is needed to address this problem, since the observed intensities of molecular emission depend on a complex competition between radiative and collisional processes. Moreover, the excitation of $\mathrm{H}_{2} \mathrm{O}$ differs from that of other molecules, since both collisions and infrared radiation from warm dust influence the level populations (Takahashi et al. 1983). The intent of this work is to show that it is not a straightforward matter to retrieve accurate information, e.g., column density, from single transition 
observations of $\mathrm{H}_{2} \mathrm{O}$ due to the complex level structure of this molecule.

\section{Basic model description}

The results presented here were obtained by applying the numerical code of Poelman \& Spaans $(2005,2006)$. The interested reader is referred to these papers for a description of the underlying algorithms. The radiative transfer of $\mathrm{o} / \mathrm{p}-\mathrm{H}_{2} \mathrm{O}$ is solved by means of a multi-zone escape probability method in three dimensions. By using a multi-zone formalism, the medium is divided into different zones, i.e., gridcells, each with a value for the abundance of the species (e.g., $\mathrm{H}_{2} \mathrm{O}$ ), the density of the medium, and the temperature of gas and dust. Besides this, the cloud is characterized by a fixed total column density. The statistical equilibrium equation for a multilevel sytem can be written as

$\sum_{i>j}\left(n_{i} A_{i j}+n n_{i} \gamma_{i j}+n_{i} B_{i j} J\right)=\sum_{i<j}\left(n n_{i} \gamma_{i j}+n_{i} B_{i j} J\right)$,

where the radiative de-excitation is given by the Einstein $A$ coefficients, $B_{i j}$ are the Einstein coefficients for absorption and stimulated emission, $\gamma_{i j}$ are the collisional rate coefficients, $J$ the mean photon radiation field, $n_{i}$ the population density of the $i$ th level, and $n$ the density of the medium. The level populations depend on the radiation field, while the radiation field depends on the level populations everywhere. By introducing the concept of a multi-zone escape probability (Poelman \& Spaans 2005, 2006) the statistical equilibrium equations are decoupled from the radiative transfer equations. Equation (1) can be rewritten as

$\sum_{i>j}\left(n_{i} A_{i j} \beta\left(\tau_{i j}\right)+n n_{i} \gamma_{i j}\right)=\sum_{i<j}\left(n n_{i} \gamma_{i j}\right)$,

because the net absorptions, corrected for stimulated emission, are equal to those photons that do not escape; i.e.,

$\left(n_{j} B_{j i}-n_{i} B_{i j}\right)_{i>j} J=\sum_{i>j} n_{i}\left[1-\beta\left(\tau_{i j}\right)\right] A_{i j}$,

where $\beta(\tau)$ is the probability that a photon formed at optical depth $\tau$ in a certain direction escapes the cloud along that direction. Therefore,

$n_{\mathrm{cr}}=\frac{\sum_{i>j} \beta\left(\tau_{i j}\right) A_{i j}}{\sum_{i>j} \gamma_{i j}}$.

Note that because of the large Einstein $A$ coefficients of the water molecule, critical densities are on the order of $10^{8}-10^{9} \mathrm{~cm}^{-3}$ in the optically thin case.

The background radiation field $P\left(v_{i j}\right)$ in the code consists of two terms: the $2.7 \mathrm{~K}$ microwave background and the infrared emission of dust at a temperature $T_{\mathrm{d}}$. This is

$P\left(v_{i j}\right)=B\left(v_{i j}, T=2.7 \mathrm{~K}\right)+\left(1-\mathrm{e}^{-\tau_{\text {dust }}}\right) B\left(v_{i j}, T_{\mathrm{d}}\right)$.

The intensity of transition $i \rightarrow j(i>j)$ is then given by

$I_{i j, \text { total }}=\frac{1}{4 \pi} \int_{0}^{r} \Lambda_{i j, \text { local }} \mathrm{d} r$

with

$\Lambda_{i j, \text { local }}=n_{i} A_{i j} h v_{i j} \beta\left(\tau_{i j}\right)\left\{\left[S\left(v_{i j}\right)-P\left(v_{i j}\right)\right] / S\left(v_{i j}\right)\right\}$,

$S\left(v_{i j}\right)$ is the source function at frequency $v_{i j}$.
Collisional rate coefficients for inelastic collisions between $\mathrm{o} / \mathrm{p}-\mathrm{H}_{2} \mathrm{O}$ and $\mathrm{He}$ (Green et al. 1993) and for collisions between o/p- $\mathrm{H}_{2} \mathrm{O}$ and both $\mathrm{o}-\mathrm{H}_{2}$ and $\mathrm{p}-\mathrm{H}_{2}$ (Phillips et al. 1996) are adopted. We adopt the expression for the ortho-to-para ratio (OPR) of $\mathrm{H}_{2}$, in thermal equilibrium, defined by

$\mathrm{OPR}=\frac{\left(2 I_{\mathrm{o}}+1\right) \sum(2 J+1) \exp \left(-\frac{E_{\mathrm{o}}\left(J, K_{\mathrm{a}}, K_{\mathrm{c}}\right)}{k T}\right)}{\left(2 I_{\mathrm{p}}+1\right) \sum(2 J+1) \exp \left(-\frac{E_{\mathrm{p}}\left(J, K_{\mathrm{a}}, K_{\mathrm{c}}\right)}{k T}\right)}$,

where $I_{\mathrm{o}}$ and $I_{\mathrm{p}}$ are the total nuclear spin, corresponding to whether the hydrogen nuclear spins are parallel $\left(I_{0}=1, \uparrow \uparrow\right)$ or anti-parallel $\left(I_{\mathrm{p}}=0, \uparrow \downarrow\right)$. The sum in the numerator (denominator) extends over all ortho (para) levels $\left(J, K_{\mathrm{a}}, K_{\mathrm{c}}\right)$; see Mumma et al. (1987). The code was tested extensively against (analytical) benchmark problems presented at the radiative transfer workshop held in Leiden (2004); see Poelman \& Spaans (2006). It is found that the level populations are completely consistent with the solutions of other Monte Carlo and ALI codes, as presented in van der Tak et al. (2005).

\section{Model results}

We calculate the surface brightness for the $1_{10} \rightarrow 1_{01}$ ground-state transition of ortho- $\mathrm{H}_{2} \mathrm{O}$, in the case of a homogeneous sphere and as a function of impact parameter. The density and abundance of $\mathrm{H}_{2} \mathrm{O}$ are the main parameters of the model. All the models have a constant total column density $N\left(\mathrm{H}_{2}\right)$ of $6 \times 10^{21} \mathrm{~cm}^{-2}$ through the center of the cloud, corresponding to a total $A_{\mathrm{V}}$ of $\sim 3 \mathrm{mag}$. As a result, the physical size of the cloud is inversely proportional to the density of the medium. The density ranges from $10^{4}$ to $10^{6} \mathrm{~cm}^{-3}$, thereby covering the relevant range for dense molecular clouds such as the Orion ridge. The temperatures, ranging from 30 to $50 \mathrm{~K}$ for the gas and from 15 to $50 \mathrm{~K}$ for the dust, were chosen to represent the mean observed temperatures towards star-forming molecular clouds such as the Orion ridge, which were the focus of the SWAS effort. In model I we ignore the emission from dust and CMB, whereas a dust temperature of $50 \mathrm{~K}$ is assumed in model IV, in order to assess the influence of the dust and temperature, respectively, on the excitation of the water molecule. The temperatures in model $\mathrm{V}$ are a factor of $\sim 2$ lower than in the other models since, to maintain a temperature of the gas of $50 \mathrm{~K}$ throughout the cloud, one needs a strong UV radiation field, which is not always the case. Note that the gas and dust temperatures are independent of cloud depth. The intent of this paper is to illustrate the excitation and radiative transfer effects assuming a "simple" cloud model, not to model a realistic cloud. A Galactic dust-to-gas ratio of $10^{-2}$ by mass is assumed. We adopt the dust opacities of Ossenkopf \& Henning (1994, Col. 5 of their Table 1). In all the models except for model I, the dust optical depth $\tau_{\text {dust }}$ through the center of the cloud at the frequency of the ground-state transition of o- $\mathrm{H}_{2} \mathrm{O}$, i.e., $\tau_{v=556.936 \mathrm{GHz}}$ or $\tau_{\lambda=538 \mu \mathrm{m}}$, is $10^{-3}$. Within each model the water abundance, $X\left(\mathrm{H}_{2} \mathrm{O}\right)$, ranges from $10^{-10}$ to $10^{-6}$. The parameters for the different models are shown in Table 1. Throughout the models, a velocity dispersion of $1 \mathrm{~km} \mathrm{~s}^{-1}$ is adopted, as is typical of a cloud with moderate turbulence. Note that the results presented in this paper depend on the adopted velocity dispersion. A higher (lower) velocity dispersion will decrease (increase) the optical depth for a given transition, thereby having an impact on the excitation of the molecule.

Figures $1-3$ present the basic results of this work. We plot, as a function of $N\left(\mathrm{H}_{2}\right)$, i.e., different impact parameter, the $1_{10} \rightarrow 1_{01}$ line intensity above the continuum per unit column density of 


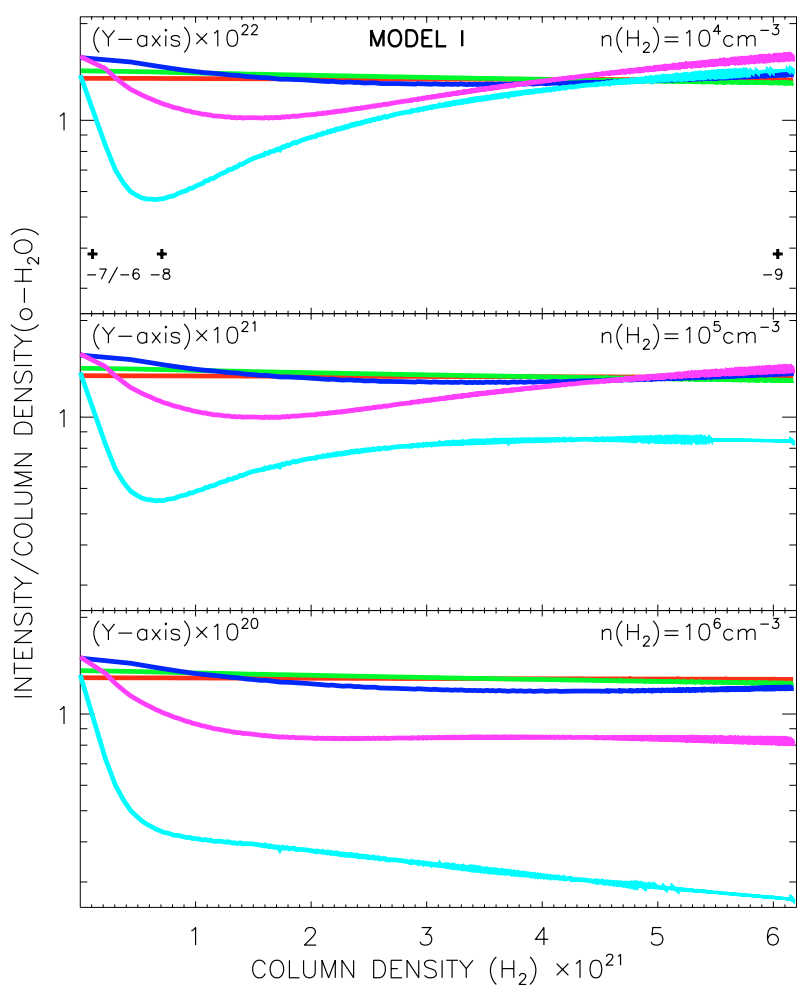

Fig. 1. The intensity of the ortho- $\mathrm{H}_{2} \mathrm{O}$ ground-state transition for a homogeneous sphere with $\mathrm{H}_{2}$ densities of $10^{4}$ (top), $10^{5}$ (middle), $10^{6}$ (bottom) $\mathrm{cm}^{-3}$ and temperature of the gas of $50 \mathrm{~K}$. The dust emission, as well as the CMB radiation, is ignored (i.e., model I). In every case, the total column density $\left(\mathrm{H}_{2}\right)$ is kept constant. Lines are plotted for an abundance of $\mathrm{H}_{2} \mathrm{O}$, relative to $\mathrm{H}_{2}$, of $10^{-10}$ (red), $10^{-9}$ (green), $10^{-8}$ (dark blue), $10^{-7}$ (purple), and $10^{-6}$ (light blue) as a function of $\mathrm{H}_{2}$ column density along the line of sight, where $2 \times 10^{19} \mathrm{~cm}^{-2}$ is at the edge, and $6 \times 10^{21} \mathrm{~cm}^{-2}$ through the center of the cloud. The $Y$-axis is in units of $\mathrm{erg} \mathrm{s}^{-1} \mathrm{sr}^{-1}$. The position of the $\tau=1$ surface is displayed with a cross for $X\left(\mathrm{H}_{2} \mathrm{O}\right)=10^{-9}-10^{-6}$.

Table 1. Model parameters.

\begin{tabular}{lcccccc}
\hline \hline Model & $\begin{array}{c}n\left(\mathrm{H}_{2}\right) \\
{\left[\mathrm{cm}^{-3}\right]}\end{array}$ & $\begin{array}{c}\text { Size } \\
{[\mathrm{pc}]}\end{array}$ & $X\left(\mathrm{H}_{2} \mathrm{O}\right)^{a}$ & $\begin{array}{c}N\left(\mathrm{H}_{2}\right)^{b} \\
{\left[\mathrm{~cm}^{-2}\right]}\end{array}$ & $\begin{array}{c}T_{\text {gas }} \\
{[\mathrm{K}]}\end{array}$ & $\begin{array}{c}T_{\text {dust }} \\
{[\mathrm{K}]}\end{array}$ \\
\hline I & $10^{4}-10^{6}$ & $0.002-0.2$ & $10^{-10}-10^{-6}$ & $6 \times 10^{21}$ & 50 & no \\
II & $10^{4}-10^{6}$ & $0.002-0.2$ & $10^{-10}-10^{-6}$ & $6 \times 10^{21}$ & 50 & 15 \\
III & $10^{4}-10^{6}$ & $0.002-0.2$ & $10^{-10}-10^{-6}$ & $6 \times 10^{21}$ & 50 & 25 \\
IV & $10^{4}-10^{6}$ & $0.002-0.2$ & $10^{-10}-10^{-6}$ & $6 \times 10^{21}$ & 50 & 50 \\
V & $10^{4}-10^{6}$ & $0.002-0.2$ & $10^{-10}-10^{-6}$ & $6 \times 10^{21}$ & 30 & 15 \\
\hline
\end{tabular}

${ }^{a}$ Abundance of $\mathrm{H}_{2} \mathrm{O} ;{ }^{b}$ total column density through the center.

o- $\mathrm{H}_{2} \mathrm{O}$, see Eq. (6). The results for model I, in which we ignore the contribution of dust and $\mathrm{CMB}$ on the excitation of the water molecule are displayed in Fig. 1. The position of the $\tau=1$ surface is denoted by a cross for $X\left(\mathrm{H}_{2} \mathrm{O}\right)=10^{-9}-10^{-6}$. Models II, III, and IV behave in a similar way as in model I in the case $n\left(\mathrm{H}_{2}\right) \geq 10^{5} \mathrm{~cm}^{-3}$. For this reason, we only plot the outcome in the low density case for models II, III, and IV in Fig. 2. The results for model $\mathrm{V}$ are plotted in Fig. 3.

The following trends can be identified, which will be discussed in Sect. 4. First, in the case of $n\left(\mathrm{H}_{2}\right) \geq 10^{5} \mathrm{~cm}^{-3}$ and $X\left(\mathrm{H}_{2} \mathrm{O}\right) \lesssim 10^{-8}$, i.e., $\tau<10$, a linear relationship holds between the number of photons escaping the cloud and the impact parameter, i.e., $I / N_{\mathrm{H}_{2} \mathrm{O}}$ is constant. However, this relationship breaks down at a high optical depth, i.e., $\mathrm{H}_{2} \mathrm{O}>10^{-8}$, for all

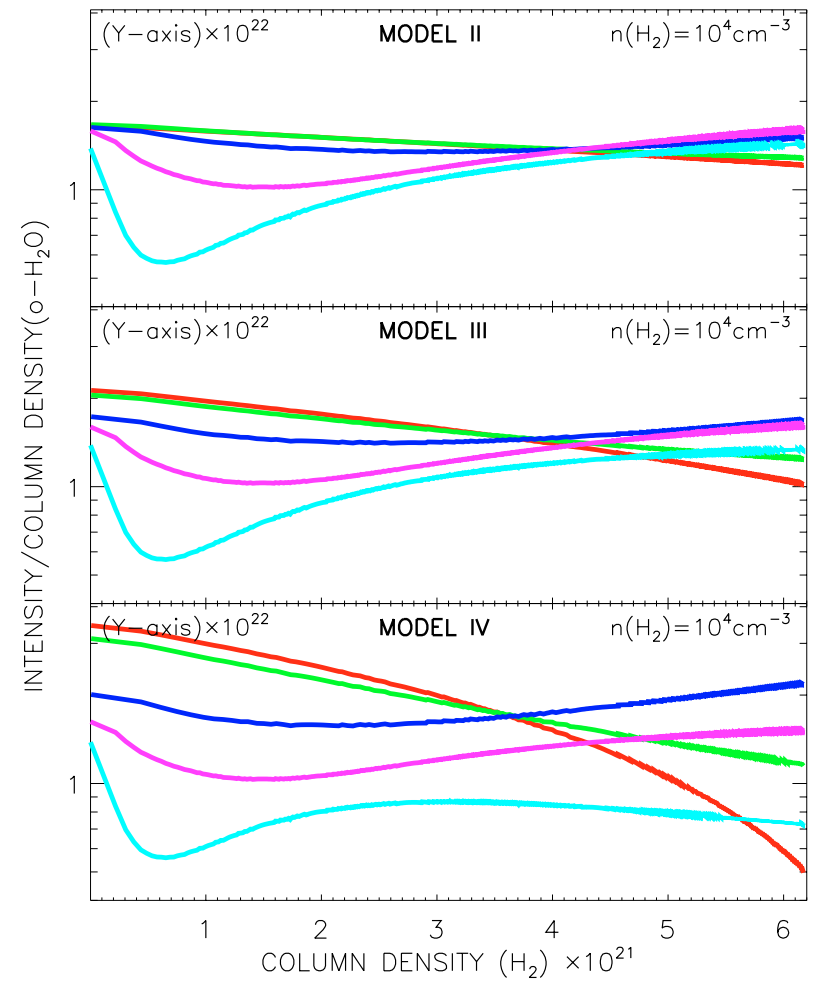

Fig. 2. The intensity of the ortho- $\mathrm{H}_{2} \mathrm{O}$ ground state transition for a homogeneous sphere with density $\left(\mathrm{H}_{2}\right)$ of $10^{4} \mathrm{~cm}^{-3}$ for model II (top), III (middle), and IV (bottom). Lines are plotted for an abundance of $\mathrm{H}_{2} \mathrm{O}$, relative to $\mathrm{H}_{2}$, of $10^{-10}$ (red), $10^{-9}$ (green), $10^{-8}$ (dark blue), $10^{-7}$ (purple), and $10^{-6}$ (light blue) as a function of $\mathrm{H}_{2}$ column density along the line of sight, where $2 \times 10^{19} \mathrm{~cm}^{-2}$ is at the edge, and $6 \times 10^{21} \mathrm{~cm}^{-2}$ through the center of the cloud. The $Y$-axis is in units of $\mathrm{erg} \mathrm{s}^{-1} \mathrm{sr}^{-1}$.

the models. Second, in all the models except model I, absorption occurs in the low density case when abundances are low, i.e., $X\left(\mathrm{H}_{2} \mathrm{O}\right)<10^{-8}$, which becomes more apparent as the dust temperature increases. However, the amount of absorption is moderate as the self-reversal in the center of the line is small. Third, when the $\mathrm{H}_{2} \mathrm{O}$ abundance exceeds $10^{-7}$, the ratio of the intensity to the column density decreases near the edge of the cloud $\left(N\left(\mathrm{H}_{2}\right) \sim 5 \times 10^{20} \mathrm{~cm}^{-2}\right)$ in all the models. Fourth, for high optical depth, i.e., $X\left(\mathrm{H}_{2} \mathrm{O}\right) \gtrsim 10^{-7}$, and $n\left(\mathrm{H}_{2}\right)=10^{6} \mathrm{~cm}^{-3}, I / N_{\mathrm{H}_{2} \mathrm{O}}$ decreases with increasing column density. Fifth, lowering the gas and dust temperatures by a factor of $\sim 2$ (model V) does not lead to significant differences in the shapes of the curves. That is, models II-IV and model V experience similar complicating radiative transfer effects.

\section{Discussion}

The asymmetry of the water molecule causes the rotational levels to split into a number of different ladders, so called " $K$-ladders", characterized by different values of the projection of the angular momentum onto the principal axes of the molecule $\left(J_{K_{-} K_{+}}\right)$. Radiative transitions occur rapidly between levels in each ladder but are much slower between levels in different ladders. This leads to a more complex spectrum than linear or symmetric top molecules, e.g., $\mathrm{CO}, \mathrm{NH}_{3}$. Hence, disentangling the different processes that contribute to the observed spectrum is not straightforward. We now describe the different effects that play a role in interpreting the figures. In this, the "edge" and "center" of the cloud refers to an impact parameter of 1 and 0 , 


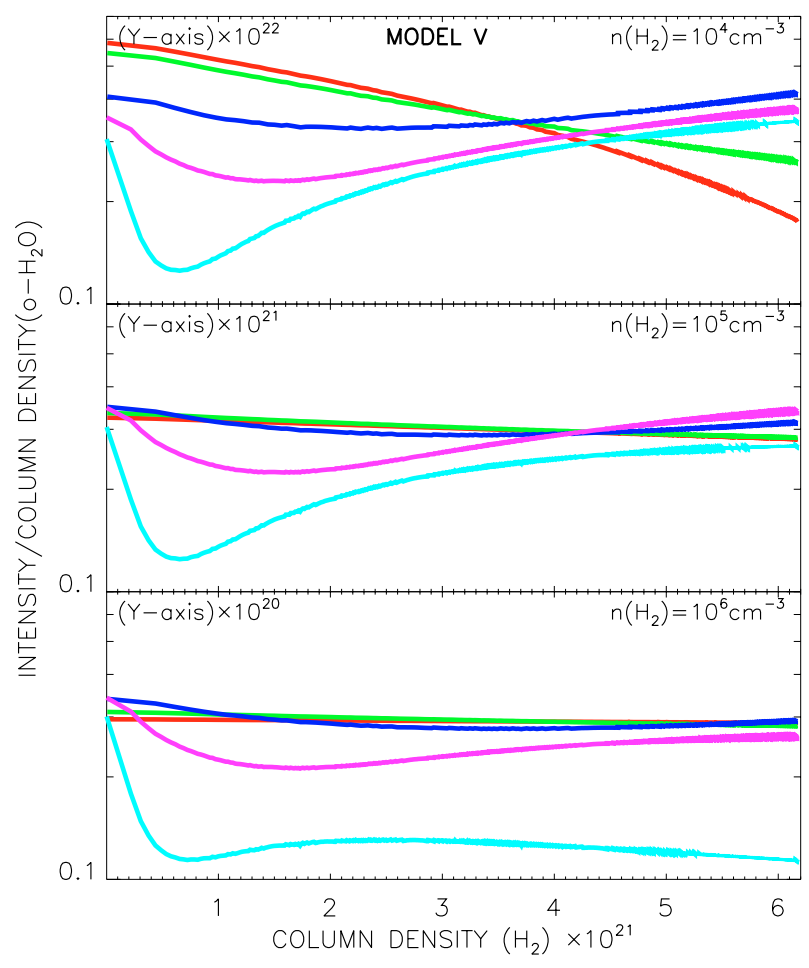

Fig. 3. The intensity of the ortho- $\mathrm{H}_{2} \mathrm{O}$ ground state transition in case of a homogeneous sphere with densities $\left(\mathrm{H}_{2}\right)$ of $10^{4}$ (top) $10^{5}$ (middle), $10^{6}$ (bottom) $\mathrm{cm}^{-3}$ and temperatures for gas and dust of $30 \mathrm{~K}$ and $15 \mathrm{~K}$, respectively (i.e., model $V$ ). In every case, the total column density $\left(\mathrm{H}_{2}\right)$ is kept constant. Lines are plotted for an abundance of $\mathrm{H}_{2} \mathrm{O}$, relative to $\mathrm{H}_{2}$, of $10^{-10}$ (red), $10^{-9}$ (green), $10^{-8}$ (dark blue), $10^{-7}$ (purple), and $10^{-6}$ (light blue) as a function of $\mathrm{H}_{2}$ column density along the line of sight, where $2 \times 10^{19} \mathrm{~cm}^{-2}$ is at the edge, and $6 \times 10^{21} \mathrm{~cm}^{-2}$ through the center of the cloud. The $Y$-axis is in units of $\mathrm{erg} \mathrm{s}^{-1} \mathrm{sr}^{-1}$.

respectively. Note that the use of spherical models leads to angular non-trivial re-distribution of line photons. The same holds for continuum photons within the line-profile frequency range.

First, one can see in Figs. 1 and 3 that the curves as function of total column density $\left(\mathrm{H}_{2}\right)$ are constant for $X\left(\mathrm{H}_{2} \mathrm{O}\right) \lesssim 10^{-8}$ and $n\left(\mathrm{H}_{2}\right) \geq 10^{5} \mathrm{~cm}^{-3}$. In this limit, collisional de-excitation and scattering effects are negligible. Eventually every photon produced in the cloud will escape the cloud with few interactions with the surrounding medium. Note that the number of scatterings $N$ to escape depends on the optical depth. In this regime $\tau \ll 1$, therefore, few photons are scattered and $N \approx \tau$. With increasing optical depth, i.e., $X\left(\mathrm{H}_{2} \mathrm{O}\right) \gtrsim 10^{-8}$, more effects have to be taken into account. All models show a drop near the edge of the cloud. Because of the increasing optical depth, line-scattering effects become important. Thus, line photons then tend to escape in the direction with the lowest optical depth rather than tangentially to the cloud surface, causing the dip near the edge. However, towards the center of the cloud, the optical depth increases with orders of magnitude. The photons will undergo numerous scatterings for $\tau \gg 1$, with $N \approx \tau^{2}$, and eventually will escape in the line wings.

Second, at densities as low as $10^{4} \mathrm{~cm}^{-3}$, and abundances not exceeding $10^{-8}$ (modest optical depth), the line is strongly subthermally excited, and radiatively colder than the dust background. Hence, the line appears in absorption. The decrease in intensity/ $\mathrm{N}\left(\mathrm{H}_{2} \mathrm{O}\right)$ shown in Fig. 2 now indicates that lines of sight through the cloud center are no longer contributing evenly to the emissivity across their entire column. The line is

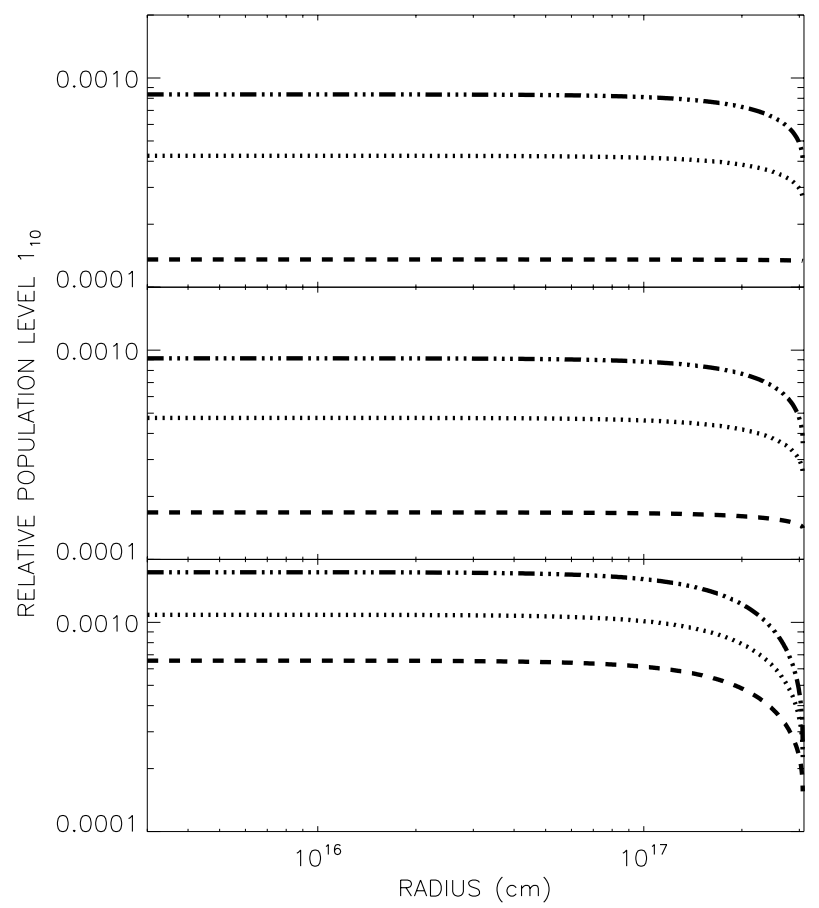

Fig. 4. The population of the $1_{01}$ level of $\mathrm{o}-\mathrm{H}_{2} \mathrm{O}$ as function of radius. The top, middle, bottom panels are the results in case $X\left(\mathrm{H}_{2} \mathrm{O}\right)=10^{-10}$, $10^{-9}, 10^{-8}$, respectively. Dashed, dotted, and dashed-dotted lines represent the results of model I, III, and IV, respectively.

not strictly in absorption yet, but it has developed an intensity dip around line center. Thus, the presence of dust causes the trend in the intensity per column density in this regime to decrease (Takahashi et al. 1983). This behavior is not seen in case $n\left(\mathrm{H}_{2}\right) \gtrsim 10^{5} \mathrm{~cm}^{-3}$, as collisions are the dominant process in the excitation of the water molecule in this regime, thereby nullifying the effect of dust emission. The influence of dust on the excitation/level populations of water is plotted in Fig. 4 where the relative population of the $1_{10}$ level is displayed. One notices that level $1_{10}$ is more populated for warmer dust. In essence, dust continuum emission will tend to drive the level populations towards a Boltzmann distribution at the temperature of the dust. For a given density, the effects of radiative excitation by dust continuum emission is more pronounced for higher dust temperatures (e.g., higher continuum intensities).

Third, the effect of photon trapping is to lower the density at which LTE is approached, i.e., after each absorption, the gas has a chance to collisionally de-excite the species and return the excitation energy to the thermal bath of the gas; see Eq. (4). For optically thin gas, the critical density of the ground-state transition at $557 \mathrm{GHz}$ of $\mathrm{o}-\mathrm{H}_{2} \mathrm{O}$ is $\sim 10^{8} \mathrm{~cm}^{-3}$ at $50 \mathrm{~K}$. The optical depth through the center of the cloud varies from 0.1 to $10^{3}$ when the abundance rises from $10^{-10}$ to $10^{-6}$ in all the models. Hence, for high abundances, i.e., $X\left(\mathrm{H}_{2} \mathrm{O}\right) \gtrsim 10^{-7}$, the effective critical density drops to $10^{5}-10^{6} \mathrm{~cm}^{-3}$, since $\beta(\tau) \sim 1 / \tau$ for high optical depth. Collisional de-excitation processes then become important in the regime where $n\left(\mathrm{H}_{2}\right) \gtrsim 10^{5} \mathrm{~cm}^{-3}$ and $X\left(\mathrm{H}_{2} \mathrm{O}\right) \gtrsim 10^{-7}$. It is seen in Figs. 1 and 3 that the $I / \mathrm{N}_{\mathrm{H}_{2} \mathrm{O}}$ drops as function of impact parameter for $n\left(\mathrm{H}_{2}\right)=10^{6} \mathrm{~cm}^{-3}$ and $X\left(\mathrm{H}_{2} \mathrm{O}\right)=10^{-6}$. In this part of parameter space, collisional de-excitation is important and the probability is high that line photons are lost to the thermal bath through collisional de-excitation during one of the many scattering events. 


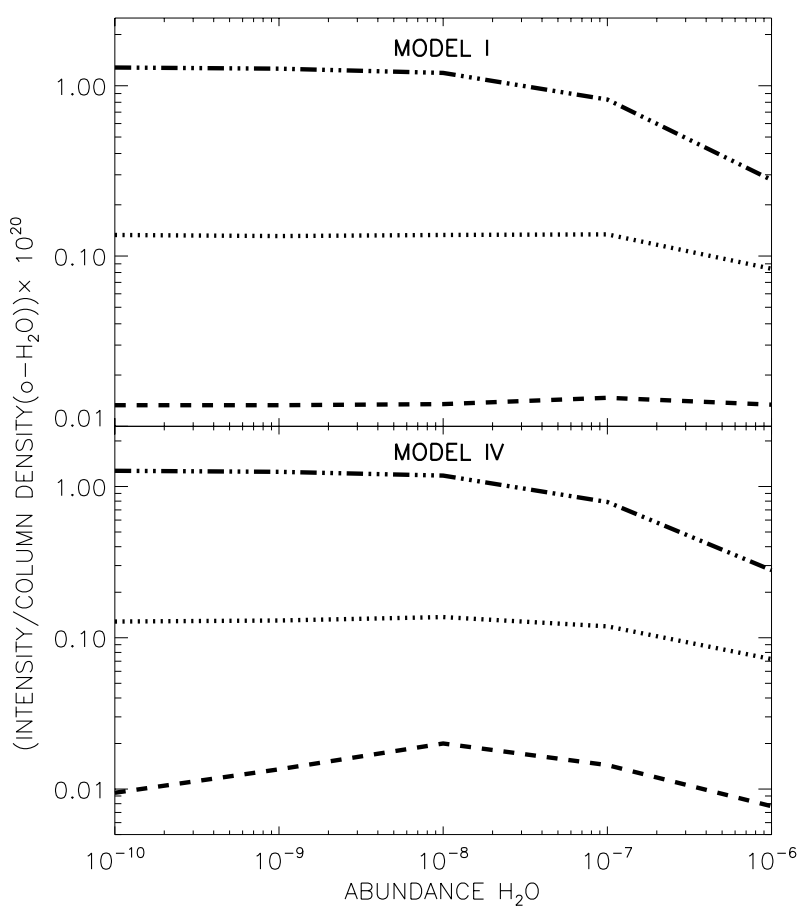

Fig. 5. Conversion of the results of models I (top) and IV (bottom) into surface area weighted values. In each panel the dashed-dotted, dotted, and dashed curves represent the results in case the density $n\left(\mathrm{H}_{2}\right)$ is $10^{6}$, $10^{5}$, and $10^{4} \mathrm{~cm}^{-3}$, respectively. The $Y$-axis is in units of $\mathrm{erg} \mathrm{s}^{-1} \mathrm{sr}^{-1}$.

Fourth, calculations are performed for model V (Fig. 3) with gas temperatures a factor of 2 lower than the temperatures used in model II. We find that the shape of the curves are not affected by such a change in gas temperature. However, it affects the distribution of the level populations and thus the absolute intensity in the lines. Hence, temperature variations cannot dispense with the radiative transfer effects studied in this work.

To summarize, we plot in Fig. 5, as a function of abundance, the average intensity emanating from the cloud for models I and IV; i.e.,

$\frac{\int I_{1_{10} \rightarrow 1_{01}}(b) 2 \pi b \mathrm{~d} b}{\int N_{\mathrm{H}_{2} \mathrm{O}}(b) 2 \pi b \mathrm{~d} b}$,

with $b$ the impact parameter. One notices a drop by a factor of $\sim 2-5$ in the case $n\left(\mathrm{H}_{2}\right)$ is $10^{5}-10^{6} \mathrm{~cm}^{-3}$, respectively. Note that with the assumption of an effectively optically thin line one would underestimate the water column density by these same factors.

\section{Astrophysical implications}

The intensity of the ground-state transition of $\mathrm{o}-\mathrm{H}_{2} \mathrm{O}$ is driven by a combination of the ambient gas and dust temperatures on the one side and by the density of the surrounding medium on the other. It is this interplay, together with the complex structure of the molecule, that drives the level populations. To interpret existing SWAS and future HIFI data, a clear sense of the information content of the water lines is needed.

SWAS observations of the lowest rotational transition

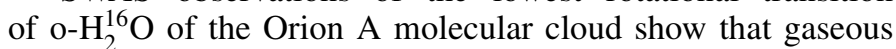
water correlates much better with the near surface tracer $\mathrm{CN}$ than with the volume tracer $\mathrm{C}^{18} \mathrm{O}$, as presented in Melnick \& Bergin (2005). Through these observations, in which it is assumed that the ground-state transition of ortho- $\mathrm{H}_{2} \mathrm{O}$ is effectively optically thin, one concludes that water is a surface tracer. This is plausible from a chemical point of view in which photo-dissociation destroys the water molecule near the surface. Farther inwards the cloud the water abundance reaches its equilibrium value through photodesorption of $\mathrm{H}_{2} \mathrm{O}$-ice and photodestruction of $\mathrm{H}_{2} \mathrm{O}$-gas until it freezes-out onto dust grains deeper into the cloud. However, we have shown (Fig. 5) that the effectively optically thin assumption no longer holds for $\tau>10$ and $n>10^{5} \mathrm{~cm}^{-3}$. Hence, under these conditions one is limited to observing the " $\tau=10$ " surface and cannot use water to trace the cloud's volume, even when it is present, i.e., not frozen out (Cernicharo, private communication). Therefore, as $\mathrm{CN}$ is a surface tracer and the water intensity originates from a layer of gas with an optical thickness of 1 - depending on local excitation conditions this layer is a surface layer - the $\mathrm{CN}$ intensity correlates much better with the $\mathrm{H}_{2} \mathrm{O}$ intensity and not with the volume tracer $\mathrm{C}^{18} \mathrm{O}$. Thus, the anti-correlation of $\mathrm{H}_{2} \mathrm{O}$ with $\mathrm{C}^{18} \mathrm{O}$ is partly due to optical depth effects, and is not neccesarily a result of chemical changes. As a consequence, the presence of water past the $\tau=1$ surface cannot be ruled out.

We would also like to point out here that the most interesting aspect of the correlation of the water line intensity with the $\mathrm{CN}$ line intensity is that both are observed to vary by a factor $\sim 100$. Theoretically, the $\mathrm{CN}$ abundance is expected to scale with density squared (Boger \& Sternberg 2005), indicating the importance of density variations over the Orion molecular cloud. Given the results presented in this paper, we surmise that these density variations will hamper the interpretation of the water observations.

In order to deduce the total water column along the line of sight, additional information is needed from other - effectively optically thin- lines, which will be observed with future missions such as Herschel/HIFI.

Acknowledgements. We are grateful to Ted Bergin and Gary Melnick for sending an early version of their paper (Melnick \& Bergin 2005). We also thank Floris van der Tak for helpful discussions and suggestions that have improved the paper and the anonymous referee for his/her constructive comments.

\section{References}

Bergin, E. A., Lellouch, E., Harwit, M., et al. 2000a, ApJ, 539, L147 Bergin, E. A., Melnick, G. J., Stauffer, J. R., et al. 2000b, ApJ, 539, L129 Boger, G. I., \& Sternberg, A. 2005, ApJ, 632, 302

Charnley, S. B., Rodgers, S. D., \& Ehrenfreund, P. 2001, A\&A, 378, 1024 Encrenaz, T. H., Lellouch, E., Cernicharo, J., et al. 1995, Icarus, 117, 162 Green, S., Maluendes, S., \& McLean, A. D. 1993, ApJS, 85, 181 Gurwell, M. A., Bergin, E. A., Melnick, G. J., et al. 2000, ApJ, 539, L143 Melnick, G. J., Ashby, M. L. N., Plume, R., et al. 2000, ApJ, 539, L87

Melnick, G. J. \& Bergin, E. A. 2005, Adv. Space Res., 36, 1027

Mumma, M. J., Weaver, H. A., \& Larson, H. P. 1987, A\&A, 187, 419

Ossenkopf, V., \& Henning, T. 1994, A\&A, 291, 943

Phillips, T. R., Maluendes, S., \& Green, S. 1996, ApJS, 107, 467

Plume, R., Kaufman, M. J., Neufeld, D. A., et al. 2004, ApJ, 605, 247

Poelman, D. R., \& Spaans, M. 2005, A\&A, 440, 559

Poelman, D. R., \& Spaans, M. 2006, A\&A, 453, 615

Snell, R. L., Howe, J. E., Ashby, M. L. N., et al. 2000a, ApJ, 539, L101

Snell, R. L., Howe, J. E., Ashby, M. L. N., et al. 2000b, ApJ, 539, L93

Takahashi, T., Silk, J., \& Hollenbach, D. J. 1983, ApJ, 275, 145

van der Tak, F., Neufeld, D., Yates, J., et al. 2005, in The Dusty and Molecular Universe: A Prelude to Herschel and ALMA, ed. A. Wilson, 431

Viti, S., Roueff, E., Hartquist, T. W., Pineau des Forêts, G., \& Williams, D. A. 2001, A\&A, 370, 557 